\title{
Poles, Physical Bounds and Optimal Materials Predicted with Approximated Mie Coefficients
}

\author{
Claire-Hélène Guidet, ${ }^{1,}{ }^{*}$ Brian Stout, ${ }^{1}$ Redha Abdeddaim, ${ }^{1}$ \\ AND NICOLAS BONOD, ${ }^{1, *}$
}

${ }^{1}$ Aix-Marseille Univ, CNRS, Centrale Marseille, Institut Fresnel, 13013 Marseille, France

*claire.guidet@fresnel.fr; nicolas.bonod@fresnel.fr

\begin{abstract}
Resonant electromagnetic scattering with particles is a fundamental problem in electromagnetism that has been thoroughly investigated through the excitation of localized surface plasmon resonances (LSPR) in metallic particles or Mie resonances in high refractive index dielectrics. The interaction strength between electromagnetic waves and scatterers is limited by maximum and minimum physical bounds. Predicting the material composition of a scatterer that will maximize or minimize this interaction is an important objective but its analytical treatment is challenged by the complexity of the functions appearing in the multipolar Mie theory. Here, we combine different kinds of expansions adapted to the different functions appearing in Mie scattering coefficients to derive simple and accurate expressions of the scattering electric and magnetic Mie coefficients in the form of rational functions. We demonstrate the accuracy of these expressions for metallic and dielectric homogeneous particles before deriving the analytical expressions of the complex eigen-frequencies (poles) for both cases. Approximate Mie coefficients can be used to derive in a simple but accurate expressions the complex dielectric permittivities that will yield a pole of the dipolar Mie coefficient and to ideal absorption conditions. The same expressions also predict the real dielectric permittivities that maximize (unitary limit) or minimize (anapole) electromagnetic scattering.
\end{abstract}

(C) 2021 Optical Society of America

\section{Introduction}

Localized surface plasmon resonances (LSPRs) have received strong attention over the last 20 years and plasmonics appears now as a mature technology [1-4]. LSPRs correspond to low order electric resonances, e.g. dipolar, quadrupolar electric resonances of the electric polarizability [5-7]. They can be described by scattering Mie coefficients $a_{n}$ and plasmonic resonances yield a maximum of $\left|a_{n}\right|$. More recently, attention has been focused on Mie resonances on dielectric particles made of high refractive index, typically semi-conductors ( $\mathrm{Si}, \mathrm{GaAs}, \mathrm{Ge}, \ldots)$ in the visible or near infrared [8-10], or ceramics, water and other materials in microwaves [11-14]. Mie resonances in dielectric particles triggered a huge interest because they intrinsically feature both electric and magnetic resonances [15-19]. While spherical subwavelength sized metallic particles can be described by electric scattering Mie coefficients, $a_{n}$, Mie resonances in dielectric particles must be described by both electric and magnetic Mie coefficients $a_{n}$ and $b_{n}$ [20]. The interplay between electric and magnetic resonances offered novel opportunities to tailor light properties in the framework of optical antennas or metasurfaces [15,21-23]. LSP and Mie resonances both correspond to a pole of a given multipolar order of a scatterer [24].

The Frölich condition corresponds to a zero of the numerator of the polarizability expression [1, 25 ] in the case of a quasi-static approximation. This approximation is used when the ratio between the size of the scatterer and the wavelength tends toward zero. In this case, the pole of the electric polarizability arises when $\epsilon_{s}=-2 \epsilon_{b}$ with $\epsilon_{s}$ and $\epsilon_{b}$ denoting the dielectric permittivities of the particle and background respectively $[1,3,25,26]$. The quasi-static expression does not fulfill the optical theorem and a radiative correction must be included in the polarizability expression [27]. Even if this correction slightly complicates the Frölich condition, it shows that the resonance 
results from a difference of sign between the real parts of the dielectric permittivities of the particle and background. In the case of Mie resonances in dielectrics, Frölich conditions cannot be applied since the real parts of $\epsilon_{b}$ and $\epsilon_{s}$ are both positive. It turns out that the quasi-static approximation, even when associated with a radiative correction term, cannot simply predict resonances occurring in subwavelength sized dielectric scatterers. That is the reason why Mie resonances in dielectrics are usually identified as the maximum of the electric and magnetic Mie coefficients calculated thanks to full numerical calculations. A major difficulty for simply describing dielectric Mie resonances is that the Taylor expansions of the functions appearing in the electric Mie scattering coefficients do not predict accurately the resonance even when a high number of terms are included in the expansion [28, 29].

Here, we show how to derive simple and accurate expressions of both electric and magnetic Mie resonances that allow for extending Frölich conditions in dielectrics. Frölich expressions are conventionally obtained by cancelling the denominator of the Mie coefficient and we show their accuracy for plasmonic particles but more importantly for dielectric particles. We thoroughly analyze the different functions of the parameter sizes $z_{s}=k R$ and $z_{0}=k_{0} R$ that appear in the Mie scattering coefficients, where $R$ is the radius of the spherical scatterer, $k=2 \pi / \lambda, k_{0}=2 \pi / \lambda_{0}$ ( $\lambda_{0}$ is the wavelength in vacuum and $\lambda$ is the wavelength in the medium of relative dielectric permittivity $\epsilon$ and $z_{s}=z_{0} \sqrt{\epsilon}$ ). By following two different methods for electric and magnetic coefficients, we derive simplified and accurate expressions of the electric and magnetic dipolar and quadrupolar Mie coefficients and polarizabilities. We assess the validity of these expressions in the case of plasmonic and dielectric scatterers. All the expressions are described by rational functions that allow us to easily extract the poles, zeros and optimal resonances that correspond respectively to a zero of the numerator, a zero of the denominator and a unitary Mie scattering coefficient. We study and plot the trajectory of the poles in the complex plane with respect to the dielectric permittivity of the scatterer. In a second step, the approximate expressions are used to derive in a simple way the physical bounds of the resonant light scattering with both dielectric and metallic particles, i.e. the unitary limit, the non-radiative anapole condition and the ideal absorption.

\section{Approximate expressions of the Mie coefficients}

\subsection{Simplified expressions of special functions}

In this section, we aim at finding the best expansion for the different functions appearing in the Mie coefficients. It is convenient to retrieve the expression of these coefficients in the framework of the scattering $T$-matrix that links the scattered field with the incident field. For a given multipolar order $n$, the electric $(e)$ and magnetic $(h)$ Mie coefficients can be straightforwardly obtained thanks to the diagonal elements of the $T_{n}^{(e)}$ and $T_{n}^{(h)}$ matrix coefficients: $a_{n}=-T_{n}^{(e)}$ and $b_{n}=-T_{n}^{(h)}$. The complete expression of the electric and magnetic Mie coefficients can be cast under the form $[20,30]$ :

$$
\begin{aligned}
& a_{n}\left(z_{0}\right)=\frac{j_{n}\left(z_{0}\right)}{h_{n}^{+}\left(z_{0}\right)} \frac{\epsilon \varphi_{n}^{1}\left(z_{0}\right)-\varphi_{n}^{1}\left(z_{s}\right)}{\epsilon \varphi_{n}^{+}\left(z_{0}\right)-\varphi_{n}^{1}\left(z_{s}\right)}, \\
& b_{n}\left(z_{0}\right)=\frac{j_{n}\left(z_{0}\right)}{h_{n}^{+}\left(z_{0}\right)} \frac{\mu \varphi_{n}^{1}\left(z_{0}\right)-\varphi_{n}^{1}\left(z_{s}\right)}{\mu \varphi_{n}^{+}\left(z_{0}\right)-\varphi_{n}^{1}\left(z_{s}\right)} .
\end{aligned}
$$

In these expressions, $\epsilon=\epsilon_{s} / \epsilon_{b}$ describes the ratio between the dielectric permittivities of the scatterer $\epsilon_{s}$ and background $\epsilon_{b}$. In the following and for the sake of clarity, $\epsilon$ will be called the dielectric permittivity of the scatterer which requires $\epsilon_{b}=1$, but it should be noticed that it is a more general parameter that describes the ratio between the permittivities of the scatterer and background. $\mu$ represents the relative magnetic permeability that is considered in this study equal to $1, \mu=1 . j_{n}(z)$ and $h_{n}^{+}(z)$ represent respectively the spherical Bessel function and the spherical 
incoming Hankel function. $\varphi_{n}^{1}(z)$ is the spherical reduced logarithmic derivative Ricatti-Bessel function and is equal to $\varphi_{n}^{1}(z)=\frac{\left(z j_{n}(z)\right)^{\prime}}{j_{n}(z)} \cdot \varphi_{n}^{+}(z)$ is the spherical reduced logarithmic derivative Ricatti-Hankel and $\varphi_{n}^{+}(z)=\frac{\left(z h_{n}^{+}(z)\right)^{\prime}}{h_{n}^{+}(z)}$. The electric (e) and magnetic (h) polarizabilities $\alpha_{n}^{(e, h)}$ of order $n$ can be simply obtained from the electric and magnetic Mie coefficients of the same order $n$ [19]:

$$
\begin{array}{rlrl}
\alpha_{1}^{e} & =a_{1} \frac{i 6 \epsilon \pi}{k^{3}}, & \alpha_{2}^{e}=a_{2} \frac{i 130 \epsilon \pi}{k^{5}} \\
\alpha_{1}^{h}=b_{1} \frac{i 6 \pi}{k^{3}}, & \alpha_{2}^{h}=b_{2} \frac{i 40 \pi}{k^{5}}
\end{array}
$$

Deriving analytic poles and zeros from the Mie coefficients requires rational functions from which it will be easy to determine the zero of the denominator or numerator. For that purpose, we derive analytical expressions of the Ricatti-Bessel functions of the first kind $\varphi_{n}^{1}\left(z_{0}\right), \varphi_{n}^{1}\left(z_{s}\right)$, and the Ricatti-Hankel function $\varphi_{n}^{+}\left(z_{0}\right)$. The Taylor expansion of $\varphi_{n}^{1}\left(z_{0}\right)$ can be used for subwavelength sized scatterers for which $z_{0}<1$ to get [31]:

$$
\varphi_{n}^{1}\left(z_{0}\right)=n+1-\frac{z_{0}^{2}}{2 n+3},
$$

The Taylor expansion of $\varphi_{n}^{1}\left(z_{s}\right)$ must be studied with care since $z_{s}$ in dielectrics is significantly larger than $z_{0}$. In particular, for the electric resonance, it has been shown a Taylor expansion of $\varphi_{n}^{1}\left(z_{s}\right)$ does not converge even at the $6^{\text {th }}$ order [29]. This weak convergence for $a_{n}$ comes from the fact that the spectral resonances for $b_{n}$ and $a_{n}$ tend respectively toward the first roots $z_{s}=r_{n-1}$ and $z_{s}=r_{n}$ of Bessel functions $j_{n-1}\left(z_{s}\right)$ and $j_{n}\left(z_{s}\right)$ respectively when $|\epsilon| \rightarrow \infty$, i.e. from the fact that the electric resonance occurs near a pole of the Ricatti-Bessel function $\varphi_{n}^{1}\left(z_{s}\right)=\frac{\left(z j_{n}\left(z_{s}\right)\right)^{\prime}}{j_{n}\left(z_{s}\right)}$ [31]. The different expressions of the $\varphi_{n}^{1}\left(z_{s}\right)$ function are plotted in Fig. 1 for $n=1$ and $n=2$. The zeros $r_{n}$ of $j_{n}\left(z_{n}\right)$ are tabulated datas, e.g. $r_{0}=3.14, r_{1}=4.49$ and $r_{2}=5.76$. They correspond to the poles of $\varphi_{n}^{1}\left(z_{s}\right)$ that are clearly observable at $z_{s}=r_{1}=4.49$, and $z_{s}=r_{2}=5.76$ in Fig. 1 where $\varphi_{n}^{1}\left(z_{s}\right)$ is plotted for $n=1$ and $n=2$. The challenge is to expand the $\varphi_{n}^{1}(z)$ function around the poles $r_{1}$ and $r_{2}$. This can be done by using a modal expansion of $\varphi_{n}^{1}\left(z_{s}\right)$ for the electric coefficients [24, 28,31,32]:

$$
\varphi_{n}^{1}\left(z_{s}\right)=n+1+\sum_{l=1}^{\infty} \frac{2 z_{s}^{2}}{z_{s}^{2}-\left(r_{n, l}\right)^{2}}
$$

with $r_{n, 1}=\pi+\frac{n \pi}{2}$. In the following we will consider only the first term, $l=1$, of this expansion so that $r_{n, 1}$ will be noted $r_{n}$. This expansion takes intrinsically into account the pole of the $\varphi_{n}^{1}(z)$ function at $z_{s}=r_{n}$. The convergence of this expansion is therefore much faster and very suitable to model resonances since only one term is enough to get a Lorentzian function. In this work, the objective is to reduce first order Mie coefficients to simple rational functions. That is the reason why we consider the first term of this expansion, even if the remaining terms $l=[2 ;+\infty]$ can be approximated by a corrective term $2 \rho_{n}^{(e)} z_{0}^{2}$ with $\rho_{n}^{(e)}=\frac{1}{r_{n}^{2}}-\frac{1}{2(2 n+3)}$ [31]. Eq. 4 reduces to a rational function when the first term of the sum is taken into account:

$$
\begin{gathered}
\varphi_{n}^{1}\left(z_{s}\right) \approx n+1+\frac{2 z_{s}^{2}}{z_{s}^{2}-r_{n}^{2}} \approx \frac{(n+3) z_{s}^{2}-(n+1) r_{n}^{2}}{z_{s}^{2}-r_{n}^{2}} \\
\varphi_{n}^{1}\left(z_{s}\right) \approx(n+1) \frac{\left(1-\frac{z_{s}^{2}}{q_{n}^{2}}\right)}{\left(1-\frac{z_{s}^{2}}{r_{n}^{2}}\right)}
\end{gathered}
$$



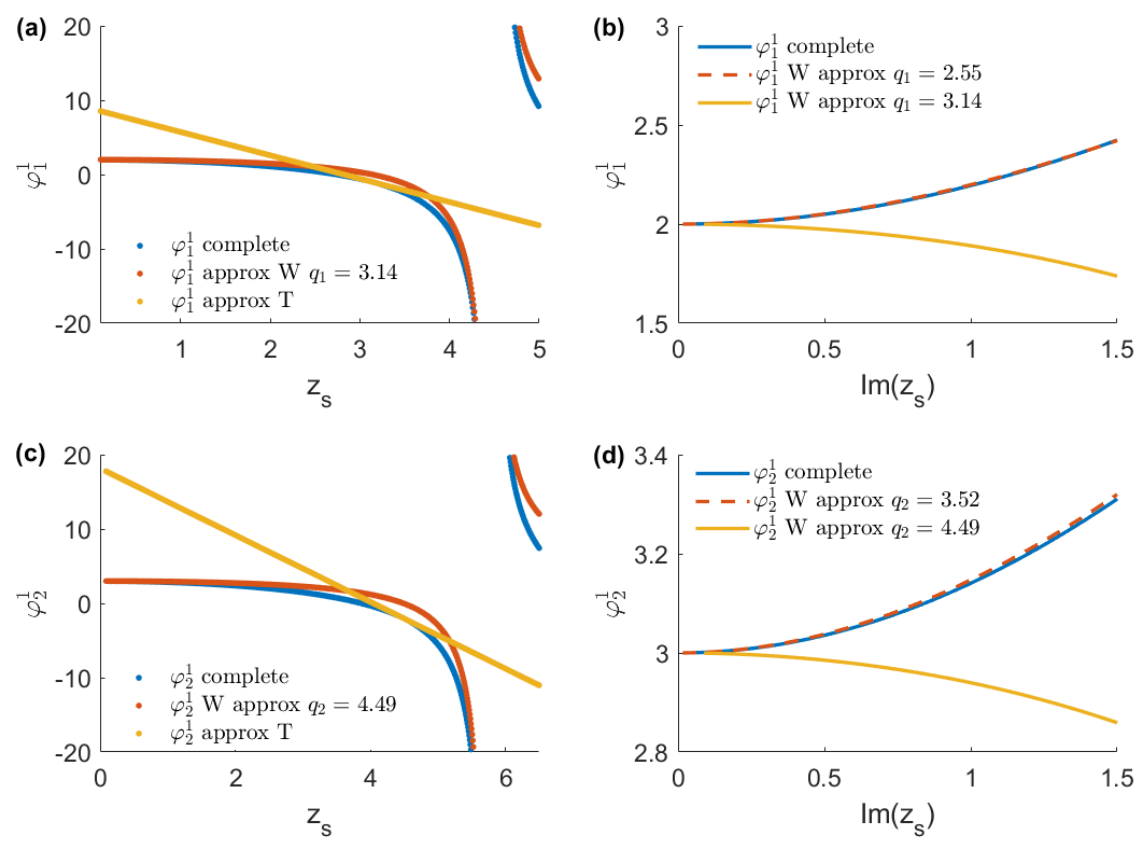

Fig. 1. Comparison between the different expansions of the function $\varphi_{n}^{1}\left(z_{s}\right)$ for the dipolar $n=1(\mathrm{a}, \mathrm{b})$ and quadrupolar $n=2(\mathrm{c}, \mathrm{d})$ cases. The calculations are displayed for a positive $(\mathrm{a}, \mathrm{c})$ and negative $(\mathrm{b}, \mathrm{d})$ dielectric permittivity. Complete calculations of $\varphi_{n}^{1}$ (blue). In (a) and (c): Taylor approximation (Approx T, yellow) calculated with Eq. 8 for $n=1$ and with Eq. 9 for $n=2$; Pole expansion (Approx W, red) calculated with Eq. 6 for $n=1$ with $q_{1}=3.14$ (a), $n=2$ with $q_{2}=4.49$ (b), $n=1$ with $q_{1}=2.55$ and $q_{1}=3.14$ (b) and $n=2$ with $q_{2}=3.52$ and $q_{2}=4.49$ (d).

with $q_{n}=r_{n} / \sqrt{\frac{n+3}{n+1}}\left(q_{1}=r_{1} / \sqrt{2} \approx 3.17 \approx r_{0}\right.$ and $\left.q_{2}=r_{2} / \sqrt{5 / 3} \approx r_{1}\right)$. We plotted in Fig. 1(a-d) the function $\varphi_{n}^{1}\left(z_{s}\right)$ calculated with Eq. 6 . The real part of the dielectric permittivity being negative for metals and positive for dielectrics respectively, we plot in Fig. 1 the function $\varphi_{n}^{1}\left(z_{s}\right)$ with respect to $z_{s}=2 \pi \sqrt{\epsilon} R / \lambda_{0}$ for dielectrics and to $\operatorname{Im}\left(z_{s}\right)$ for metals. This means that we plot the function $\varphi_{n}^{1}\left(z_{s}\right)$ for metals and dielectrics separately but with a continuous transition between these two regimes since we can observe the continuity of the $\varphi_{n}^{1}\left(z_{s}\right)$ function at $z_{s}=0$. We first consider the values $q_{1}=r_{0}=\pi$ and $q_{2}=r_{1}$ to plot the function $\varphi_{n}^{1}\left(z_{s}\right)$ with Eq. 6 and it can be observed that these values permit to match the complete function for dielectrics in Fig. 1(a,c), while they fail to retrieve the feature of the complete function $\varphi_{n}^{1}\left(z_{s}\right)$ for metals in Fig. 1(b,d). This result shows that the terms that are not taken into account in expansion Eq. 4 $(l \geq 2)$ have a larger contribution for metals than for dielectrics. Let us emphasize that this value of $q_{n}$ is obtained when considering the first term only in the infinite sum of Eq. 4. A modification of this initial value $q_{n}=r_{n} / \sqrt{\frac{n+3}{n+1}}$ can be considered to partially take into account the higher order terms in Eq. 4. A tuning of the $q_{n}$ parameter is therefore performed to improve the match between the approximate and rigorous expressions of $\varphi_{n}^{1}\left(z_{s}\right)$ and we found that $q_{1}=2.55$ and $q_{2}=3.52$ offer much better results in the case of metallic scatterers as it can be observed in Fig. 1(b,d)).

Magnetic resonances are located near $z_{s}=r_{n-1}$ when $\epsilon \gg 1$ [31]. They are therefore far from the pole of $\varphi_{n}^{1}(z)$ located at $z_{s}=r_{n}$. Taylor expansions can thereby be safely used for the 
magnetic coefficients $b_{n}$. However, while Taylor expansion is classically expanded near $z_{s}=0$ in the case of subwavelength scatterers, it is judicious to perform this Taylor expansion at the first order at the position of the resonance, $z_{s}=r_{n-1}$, to improve the convergence [31]:

$$
\varphi_{n}^{1}\left(z_{s}\right)=-n-r_{n-1}\left(z_{s}-r_{n-1}\right)
$$

This expression gives for the first two multipoles $n=1$ and $n=2$ :

$$
\begin{aligned}
& \varphi_{1}^{1}\left(z_{s}\right)=-1-r_{0}\left(z_{s}-r_{0}\right), \\
& \varphi_{2}^{1}\left(z_{s}\right)=-2-r_{1}\left(z_{s}-r_{1}\right) .
\end{aligned}
$$

We followed different expansions, i.e. modal and Taylor expansions, to retrieve fractional functions of the electric and magnetic coefficients. These two different expansions of function $\varphi_{n}^{1}\left(z_{s}\right)$ are compared in Fig. 1 for the dipolar (top) and for the quadrupolar (bottom) cases.

We now derive the analytic expression of the second special function that appears in the expressions of $a_{n}$ and $b_{n}$ (Eqs. 1a,1b), the spherical Ricatti-Hankel incoming (+) and outgoing (-) function: $\varphi_{n}^{ \pm}=\frac{\left(z h_{n}^{ \pm}(z)\right)^{\prime}}{h_{n}^{ \pm}(z)}$ with $h_{n}^{ \pm}(z)=j_{n}(z) \pm i y_{n}(z)$, the Hankel function. $\varphi_{1}^{ \pm}(z)$ has a meromorphic expression [28]:

$$
\varphi_{1}^{ \pm}\left(z_{0}\right)= \pm i z_{0}-\frac{1}{1 \mp i z_{0}},
$$

we have to derive the meromorphic form of $\varphi_{2}^{+}\left(z_{0}\right)$. For that purpose, we use the recurrence relation:

$$
\varphi_{n}^{+}\left(z_{0}\right)=-n+z \frac{h_{n-1}^{+}\left(z_{0}\right)}{h_{n}^{+}\left(z_{0}\right)}
$$

The $h_{2}^{+}$function that appears in this recurrence expression of $\varphi_{2}^{+}\left(z_{0}\right)$ can be obtained thanks to a recursive form of the Hankel spherical function [32] :

$$
h_{n+1}^{ \pm}\left(z_{0}\right)=-h_{n-1}^{ \pm}\left(z_{0}\right)+\frac{2 n}{z_{0}} h_{n}^{ \pm}\left(z_{0}\right) .
$$

This recurrence relation expanded for $h_{2}^{+}\left(z_{0}\right)$ requires the expressions of $h_{0}^{+}\left(z_{0}\right)$ and $h_{1}^{+}\left(z_{0}\right)[28,33]$ :

$$
h_{0}^{+}\left(z_{0}\right)=\frac{1}{3}-\frac{i}{z_{0}}, h_{1}^{+}\left(z_{0}\right)=-e^{i z_{0}} \frac{z_{0}+i}{z_{0}^{2}} .
$$

The use of these two expressions in the recurrence relation, Eq. 12, leads to $h_{2}^{+}\left(z_{0}\right)=\frac{i}{z_{0}} e^{i z_{0}}(1+$ $\left.\frac{3 i}{z_{0}}-\frac{3}{z_{0}^{2}}\right)$. The two meromorphic forms of $h_{1}^{+}\left(z_{0}\right)$ and $h_{2}^{+}\left(z_{0}\right)$ allow us to find the expression of $\varphi_{2}^{+}\left(z_{0}\right)$ :

$$
\varphi_{2}^{+}\left(z_{0}\right)=-2+\frac{-z_{0}-i}{i\left(1+\frac{3 i}{z_{0}}-\frac{3}{z_{0}^{2}}\right)}
$$

The last function appearing in the Mie coefficient expression is the Bessel function $j_{n}\left(z_{0}\right)$. An interesting expansion to approximate $j_{n}\left(z_{0}\right)$ is the Pade expansion that consists of expressing a function in its rational form. We consider this expansion to expand the spherical Bessel function $j_{n}\left(z_{0}\right)$. For the first two orders, $n=1$ and $n=2$, we obtain $[18,34,35]$ :

$$
j_{1}\left(z_{0}\right)=\frac{z_{0}}{3\left(1+\frac{z_{0}^{2}}{10}\right)},
$$




$$
j_{2}\left(z_{0}\right)=\frac{z_{0}^{2}}{15\left(1+\frac{z_{0}^{2}}{14}\right)},
$$

The analytic expressions of the special functions derived in the previous section can now be used to expand for $n=1$ and $n=2$ the Mie coefficients $a_{n}$ and $b_{n}$ provided in Eqs. (1a,1b).

\subsection{Magnetic dipolar and quadrupolar Mie coefficients}

Let us start with the dipolar and quadrupolar magnetic Mie coefficients for which the convergence of the Taylor expansions of the special functions around the spectral positions of the resonances $z_{s}=r_{n-1}$ is fast. The use of expressions in Eq. 8,9,10,14 of the special functions in Eq. 1b allow us to get $b_{1}\left(z_{0}\right)$ and $b_{2}\left(z_{0}\right)$ :

$$
\begin{aligned}
& b_{1}\left(z_{0}\right)=\frac{-z_{0}^{3}}{3\left(z_{0}+i\right) e^{i z_{0}}\left(1+\frac{z_{0}^{2}}{10}\right)} \frac{3-\frac{z_{0}^{2}}{5}+r_{0}\left(z_{s}-r_{0}\right)}{\left(i z_{0}-\frac{1}{1-i z_{0}}\right)+1+r_{0}\left(z_{s}-r_{0}\right)}, \\
& b_{2}\left(z_{0}\right)=\frac{z_{0}^{3} e^{-i z_{0}}}{15 i\left(1+\frac{z_{0}^{2}}{14}\right)\left(1+\frac{3 i}{z_{0}}-\frac{3}{z_{0}^{2}}\right)} \frac{5-\frac{z_{0}^{2}}{7}+r_{1}\left(z_{s}-r_{1}\right)}{\frac{-z_{0}-i}{i\left(1+\frac{3 i}{z_{0}}-\frac{3}{z_{0}^{2}}\right)}+r_{1}\left(z_{s}-r_{1}\right)} .
\end{aligned}
$$

These expressions have the form of rational functions from which it will be easy to extract the poles and physical bounds.

\subsection{Electric dipolar and quadrupolar Mie coefficients}

The electric dipolar and quadrupolar Mie coefficients $a_{1}$ and $a_{2}$ are obtained thanks to the modal expansion in its approximated form in Eq. 6:

$$
\begin{aligned}
& a_{1}\left(z_{0}\right)=\frac{-z_{0}^{3}}{3\left(z_{0}+i\right) e^{i z_{0}}\left(1+\frac{z_{0}^{2}}{10}\right)} \frac{\epsilon\left(2-\frac{z_{0}^{2}}{5}\right)-2 \frac{1-\left(\frac{z_{s}}{q_{1}}\right)^{2}}{1-\left(\frac{z_{s}}{r_{1}}\right)^{2}}}{\epsilon\left(i z_{0}-\frac{1}{1-i z_{0}}\right)-2 \frac{1-\left(\frac{z_{s}}{q_{1}}\right)^{2}}{1-\left(\frac{z_{s}}{r_{1}}\right)^{2}}} \\
& a_{2}\left(z_{0}\right)=\frac{z_{0}^{3} e^{-i z_{0}}}{15 i\left(1+\frac{3 i}{z_{0}}-\frac{3}{z_{0}^{2}}\right)\left(1+\frac{z_{0}^{2}}{14}\right)} \\
& \epsilon\left(3-\frac{z_{0}^{2}}{7}\right)-3 \frac{1-\left(\frac{z s}{q_{2}}\right)^{2}}{1-\left(\frac{z_{s}}{r_{2}}\right)^{2}} \\
& \overline{\epsilon\left(-2+\frac{-z_{0}-i}{i\left(1+\frac{3 i}{z_{0}}-\frac{3}{z_{0}^{2}}\right)}\right)-3 \frac{1-\left(\frac{z_{s}}{q_{2}}\right)^{2}}{1-\left(\frac{z_{s} s}{r_{2}}\right)^{2}}}
\end{aligned}
$$

We assess the accuracy of such expressions for both plasmonic and dielectric Mie scatterers by calculating Mie coefficients with approximated (Eqs. 17-20) and complete (Eqs. 1a,1b) expressions. Mie coefficients can be calculated in the whole range of frequencies. That is the reason why we consider scatterers composed of a dielectric permittivity of $\epsilon=78.4$ (distilled water in the $\mathrm{GHz}$ spectrum), $\epsilon=16$ (semi-conductor in the visible and NIR) and a silver particle in the visible spectrum with a dielectric permittivity taken from Johnson [36]. In the case of spherical scatterers made of silver (Fig. 2(c)), we only plot the dipolar and quadrupolar electric coefficient since magnetic coefficients are negligible. The $q_{n}$ parameter used in the expressions of 


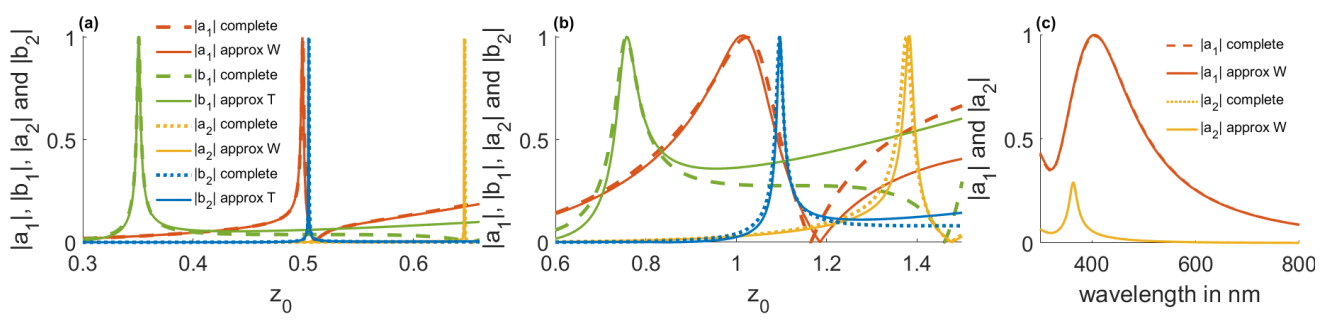

Fig. 2. Spectrum of the electric and magnetic Mie coefficients $a_{1}$ (red), $a_{2}$ (orange), $b_{1}$ (green), $b_{2}$ (blue) with respect to the parameter size $z_{0}=k R$ obtained with approximate expressions in Eqs. 17-20. Calculations performed with scatterers in air (or vacuum) of dielectric permittivity $\epsilon=78.4$ (a), $\epsilon=16$ (b), and silver (dielectric permittivity taken from Johnson [36]) (c). Full calculations : dashed lines for $n=1$ and dotted lines for $n=2$; approximate calculations: solid line. Coefficients $a_{1}$ and $a_{2}$ are calculated with Eqs. 19-20 with $q_{1}=3.14$ and $q_{2}=4.49$ for dielectrics (in (a) and (b)) and with $q_{1}=2.55$ and $q_{2}=3.52$ for metals (c). Coefficients $b_{1}$ and $b_{2}$ (in (a) and (b)) are calculated with Eqs. 17-18.

the electric Mie coefficients is taken equal to $q_{1}=r_{0}$ and $q_{2}=r_{1}$ for dielectrics and $q_{1}=2.55$ and $q_{2}=3.52$ for metals. We compare in Fig. 2 the dipolar and quadrupolar Mie coefficients obtained by using the rigorous (Eqs. 1a,1b) and approximated (Eqs. 17-20) expressions. We observe for the 4 cases that the simplified expressions permit to fully retrieve both plasmonic and dielectric Mie resonances. This high accuracy is verified for dipolar and quadrupolar approximations.

\section{Poles and physical bounds}

We now aim at benefiting from these rational formulations of dielectric polarizabilities (or equivalently first order Mie coefficients) to derive the analytical expressions of the anapoles, poles and resonances. Anapoles correspond to lower physical bounds for which distributions of currents do not yield electromagnetic scattering [37-42]. Under the assumption of subwavelength particles for which multipolar contributions are well separated spectrally, anapoles can be predicted by the zeros of the corresponding Mie coefficient. Resonances must be distinguished from the poles that correspond to a solution of Maxwell equations in absence of source, while a resonance corresponds to a maximum coupling between the excitation field and the eigen-modes and also corresponds to a maximum of the electromagnetic scattering yielded by the scatterer. This condition corresponds to the so-called unitary limit for which the norm of a multipolar Mie coefficient is equal to 1 [29]. To summarize, this section will aim at determining the zeros (anapoles), poles and optimal resonances, i.e. unitary limit of the first order Mie coefficients of subwavelength sized scatterers.

\subsection{Poles}

Poles correspond to a zero of the denominator of the rational functions describing the polarizabilities of the scatterer. They correspond to solutions in the complex $\omega$ or $z_{0}$ plane $\left(z_{s}=\sqrt{\epsilon} z_{0}=\sqrt{\epsilon} R \omega / c\right)$ of equations $\epsilon \varphi_{1}^{+}\left(z_{0}\right)-\varphi_{1}^{1}\left(z_{s}\right)=0$ and $\varphi_{1}^{+}\left(z_{0}\right)-\varphi_{1}^{1}\left(z_{s}\right)=0$ for the electric and magnetic dipolar cases respectively. These equations can be solved by using the complete and full numerical expressions of $\varphi_{1}^{+}\left(z_{0}\right)$ and $\varphi_{1}^{1}\left(z_{s}\right)$ and finding the minimum in the complex $\omega$ plane by using a Newton-Raphson iteration method.

Here, we show how to retrieve the poles analytically by cancelling the denominators of the rational approximate expressions of the electric and magnetic polarizability in Eqs. 19,17: 


$$
\begin{gathered}
\epsilon\left(i z_{0}-\frac{1}{1-i z_{0}}\right)=2 \frac{1-\left(\frac{z_{s}}{r_{0}}\right)^{2}}{1-\left(\frac{z_{s}}{r_{1}}\right)^{2}}, \\
i z_{0}-\frac{1}{1-i z_{0}}=-1-r_{0}\left(z_{s}-r_{0}\right) .
\end{gathered}
$$

that correspond to solve a $4^{\text {th }}$ and a $2^{\text {nd }}$ order differential equation:

$$
\begin{gathered}
z_{0, e}^{4}\left(-\frac{\epsilon^{2}}{r_{1}^{2}}\right)+z_{0, e}^{3}\left(-\frac{i \epsilon^{2}}{r_{1}^{2}}-\frac{2 i \epsilon}{r_{0}^{2}}\right)+z_{0, e}^{2}\left(\epsilon+\frac{\epsilon^{2}}{r_{1}^{2}}+\frac{2 \epsilon}{r_{0}^{2}}\right) \\
+z_{0, e}(2 i+i \epsilon)+(-\epsilon-2)=0 \\
z_{0, h}^{2}\left(1-i r_{0} \sqrt{\epsilon}\right)+z_{0, h}\left(r_{0} \sqrt{\epsilon}+i r_{0}^{2}\right)-r_{0}^{2}=0 .
\end{gathered}
$$

In the electric case (Eq. 23), the $4^{\text {th }}$ order polynomial equation leads to 4 solutions. Two solutions feature a positive imaginary frequency. Causality imposes negative imaginary parts of the eigen-frequencies and these 2 solutions can be safely removed [43-49]. The only two solutions with negative imaginary parts satisfy the causality principle and correspond to the first and second electric poles. These solutions can be calculated for any scatterer and material and we plot the complex eigen-frequencies with respect to the dielectric permittivity (see Fig. 3).
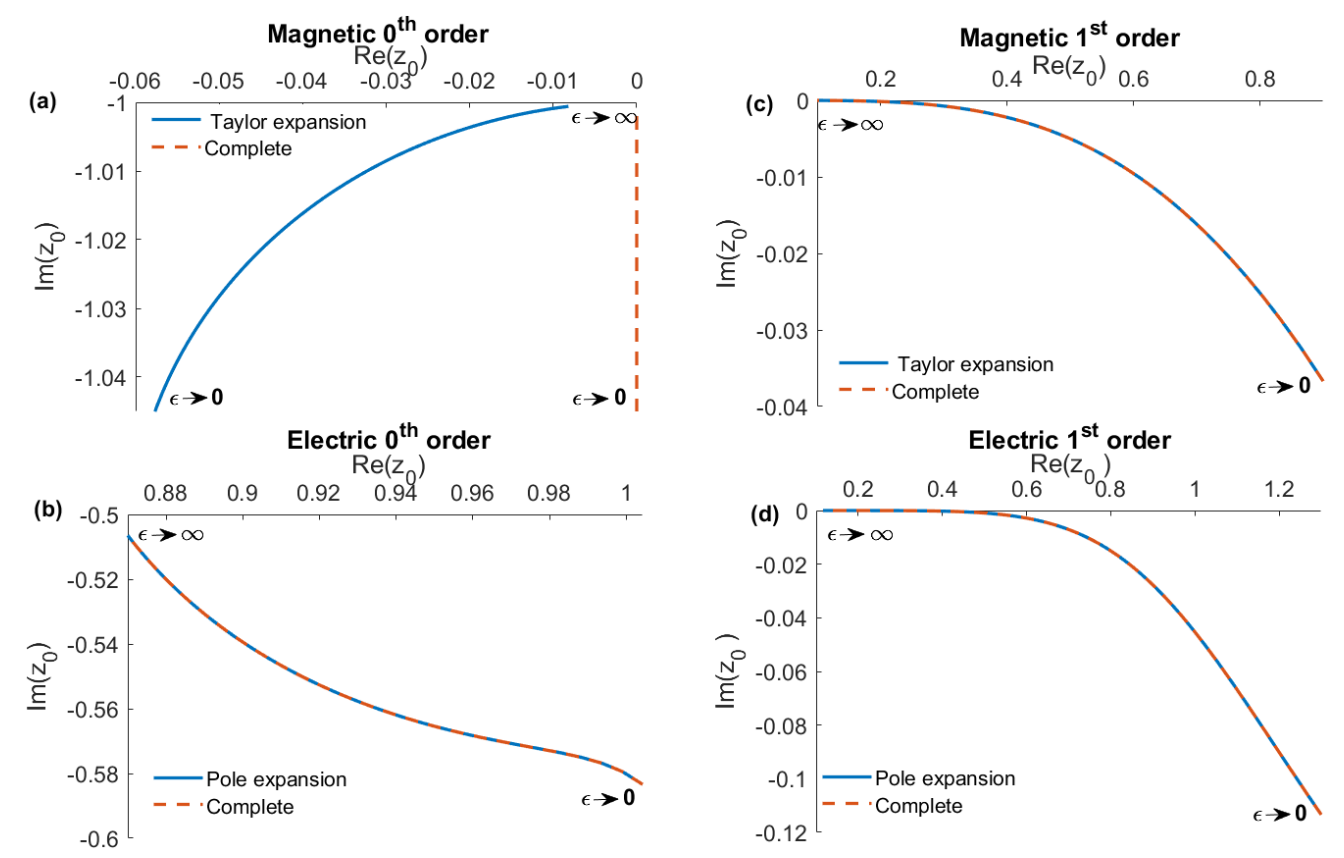

Fig. 3. Trajectory of the complex electric and magnetic poles in the complex $z_{0}$ plane when spanning the dielectric permittivity of the scatterer, $\epsilon$, from $0^{+}$to $\infty$. (a,b): fundamental pole; (c,d): first pole, (a,c): magnetic poles; (c,d) electric poles. Complete calculations (Newton-Raphson iteration method): red dashed line; approximate expressions taken from Eq. 23 (a,b) and Eq. 24 (c,d): blue full line.

The fundamental dipolar electric mode has a large negative imaginary part while the first mode has a much lower imaginary value. One can observe that the real parts decrease when the 
dielectric permittivity increases with a much larger decrease for the second mode. The decrease of the real part with $\epsilon$ is associated with a decrease of the negative part. The validity of these expressions is assessed by comparing the eigen-frequencies found by the analytic expressions and by the full numerical calculations associated with the Newton-Rhapson iteration method (see Fig. 3(a,b,c,d)). The agreement is excellent for the fundamental (see Fig. 3(a)) and 1st modes (see Fig. 3(b)) for the electric case. The method can also be applied to find the poles in the case of metals for which for $q_{1}=2.55$. In the case of a dielectric permittivity of $\epsilon=-2.4$, the the electric poles obtained from the full and approximate expressions are respectively $z_{o, p}=0.3719-i 0.059$ and $z_{o, p}=0.3710-i 0.058$. The relative error is of $0.2 \%$ and $1.69 \%$ for the real and imaginary parts. For the magnetic case (Eq. 24), the $2^{\text {nd }}$ order polynomial equation is solved analytically and the 2 roots are found from the expression of the discriminant:

$$
\Delta=\left(r_{0} \sqrt{\epsilon}+i r_{0}^{2}\right)^{2}+4\left(1-i r_{0} \sqrt{\epsilon}\right) r_{0}^{2}
$$

Each root corresponds to a pole. The 2 poles can be calculated with respect to the dielectric permittivity. We can see that the two poles feature a very different dependence with respect to the dielectric permittivity. The fundamental pole stays along the real axis with a real part $\approx 0$ while the imaginary part stays far under the real axis and at a value around -1 . The full calculations show that this pole has a real part equal to zero. Both real and imaginary parts are weakly sensitive to the dielectric permittivity. The trajectory of the second pole in the complex $z_{0}$ plane is very different: it is located just underneath the imaginary axis, the real part is strictly positive. When the dielectric permittivity increases, the real part increases while the imaginary part tends toward zero. The comparison between the approximated and full calculations shows a remarkable agreement for the first pole (see Fig. 3(a)), that drives the resonant response of the scatterer. The agreement is less favorable for the fundamental pole (see Fig. 3(c)) located on the imaginary axis but this error has less influence since this pole, far below the real axis, has less influence on the electromagnetic response of the scatterer.

A very convenient way of finding poles is to solve the roots of the denominators of the polarizability expressions with respect to the dielectric permittivity [50]. For the magnetic dipolar polarizability, one obtains a single complex solution, $\epsilon_{p, h}$, that is simply obtained with respect to the parameter size $z_{0}$ and $r_{0}=\pi$.

$$
\epsilon_{p, h}=\left(\frac{-z_{0}^{2}+r_{0}^{2}-i r_{0}^{2} z_{0}}{r_{0} z_{0}-i r_{0} z_{0}^{2}}\right)^{2}
$$

In the case of the electric dipole, one obtains a $2^{\text {nd }}$ order polynomial equation:

$$
\begin{aligned}
& \epsilon_{p, e}^{2}\left(-i \frac{z_{0}^{3}}{r_{1}^{2}}-\frac{z_{0}^{4}}{r_{1}^{2}}+\frac{z_{0}^{2}}{r_{1}^{2}}\right)+ \\
& \epsilon_{p, e}\left(i z_{0}+z_{0}^{2}-1+\frac{2 z_{0}^{2}}{r_{0}^{2}}-2 i \frac{z_{0}^{3}}{r_{0}^{2}}\right)+\left(2 i z_{0}-2\right)=0
\end{aligned}
$$

The two solutions have positive imaginary parts but real parts of opposite \pm signs. In other words, these single expressions in Eqs. 19,20 catch the poles of both localized surface plasmons in metals and Mie modes in dielectrics. In this sense, they extend the so-called Frölich conditions to dielectric scatterers.

The real and imaginary parts of $\epsilon_{p, e}$ and $\epsilon_{p, h}$, solutions of the electric and magnetic dipolar poles, are plotted in Fig. 6 in the $\mathbb{R} e(\epsilon)$ and $\operatorname{Im}(\epsilon)$ plane. In the case of negative $\mathbb{R} e(\epsilon)$ in Fig. 6 (b), we retrieve in the limit $z_{0} \rightarrow 0$ the quasi-static solution $\mathbb{R} e(\epsilon) \rightarrow-2, \operatorname{Im}(\epsilon) \rightarrow 0$. When $z_{0}$ increases, the real part decreases down to a minimum of $\mathbb{R} e(\epsilon) \approx-2.8$ before increasing back to 
$\approx-2$ for large parameter size $z_{0} \approx 1$. In the case of dielectric scatterers, $\mathbb{R} e(\epsilon)_{p,(e, h)} \rightarrow 0$ and $\operatorname{Im}(\epsilon)_{p,(e, h)} \rightarrow 0$ when $z_{0} \rightarrow 0$. We observe that losses associated with electric poles $\epsilon_{p, e}$ are generally much smaller than for magnetic poles $\epsilon_{p, h}$.

\subsection{Lower bounds: zeros and anapoles}

In this section, we aim at finding the roots $z_{0, z}$ of the numerator of the Mie coefficient expressions of $a_{1}$ and $b_{1}$. We focus on the electric anapole since they are found at much smaller parameter sizes than magnetic anapoles [42]. We benefit from the expressions of the previous section to find the roots that cancel the electric dipolar polarizability of a sub-wavelength sized scatterer. Cancelling the numerator of Eq. 19 leads to a $4^{\text {th }}$ order polynomial equation:

$$
z_{0, z}^{4}\left(\frac{\epsilon^{2}}{5 r_{1}^{2}}\right)+z_{0, z}^{2}\left(\frac{-\epsilon}{5}-\frac{2 \epsilon^{2}}{r_{1}^{2}}+\frac{2 \epsilon}{r_{0}^{2}}\right)+(2 \epsilon-2)=0
$$

This equation provides four roots:

$$
\begin{aligned}
& z_{0, z}= \pm \sqrt{\frac{-\left(\frac{-\epsilon}{5}-\frac{2 \epsilon^{2}}{r_{1}^{2}}+\frac{2 \epsilon}{r_{0}^{2}}\right) \pm \sqrt{\Delta}}{2 \frac{\epsilon^{2}}{5 r_{1}^{2}}}} \\
& \Delta=\left(\frac{-\epsilon}{5}-\frac{2 \epsilon^{2}}{r_{1}^{2}}+\frac{2 \epsilon}{r_{0}^{2}}\right)^{2}-4(2 \epsilon-2)\left(\frac{\epsilon^{2}}{5 r_{1}^{2}}\right) .
\end{aligned}
$$

All the roots are real for $\epsilon \gtrsim 6.96$, which corresponds to most of the cases considered when studying Mie resonances. The finding of real solutions means that complete zeros must be observed in the spectrum of $a_{1}$ as soon as $\epsilon \gtrsim 6.96$. We study only the two roots that provide a positive $z_{0, z}$ value. One of this solution has large values, typically $z_{0, z} \approx 3$, i.e. for scatterers much larger than the wavelength and we focus our attention on the other solution that provides solutions for subwavelength sized scatterers (see Fig. 4). It is insightful to recast Eq. 28 to get $\epsilon$ with respect to the parameter size. This expression will tell us the composition of the scatterer that cancels a scattering channel for each parameter size. Let us focus for example on the first root that provides the smallest value permittivity leading to an anapole :

$$
\begin{aligned}
& \epsilon_{z_{0}}=\frac{-\left(2-\frac{z_{0}^{2}}{5}+\frac{z_{0}^{2}}{r_{0}^{2}}\right)+\sqrt{\Delta_{z}}}{2\left(\frac{-2 z_{0}^{2}}{r_{1}^{2}}+\frac{z_{0}^{4}}{r_{1}^{2}}\right)} \\
& \Delta_{z}=\left(2-\frac{z_{0}^{2}}{5}+\frac{z_{0}^{2}}{r_{0}^{2}}\right)^{2}+8\left(\frac{-2 z_{0}^{2}}{r_{1}^{2}}+\frac{z_{0}^{4}}{r_{1}^{2}}\right) .
\end{aligned}
$$

In the case of subwavelength sized scatterers, one finds a positive and purely real dielectric permittivity, whose value strongly increases when the parameter size decreases. This means that anapoles can be found for any parameter size and for any dielectric permittivity (see Fig. 4).

\subsection{Upper Bound: Unitary Limit}

The resonant interaction between an electromagnetic wave and a scatterer is limited by physical bounds [29,51-53]. Physical bounds can be described by the maximum of the electromagnetic radiation that can be absorbed by a scatterer in a homogeneous or complex environment $[28,44$, 54-59]. Physical bounds can also be described by the maximum of electromagnetic scattering. In the framework of the multipolar theory, this limit corresponds to the maximum of electromagnetic scattering in a single channel corresponding to a given multipolar order $[29,51-53,60]$, i.e. 


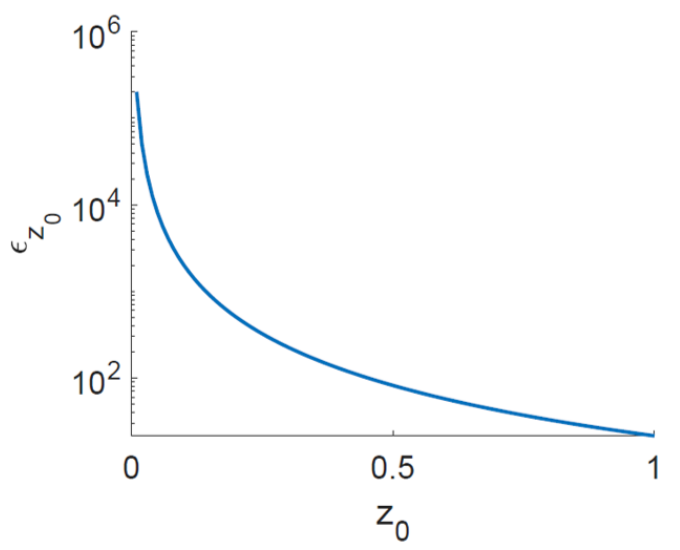

Fig. 4. Anapoles in dielectric scatterers. Dielectric permittivity, $\epsilon_{z_{0}}$ that cancels the electric dipolar coefficient $a_{1}$, calculated with Eq. 30, plotted with respect to the parameter size $z_{0}$.

when the norm of this multipolar order is equal to $1,\left|a_{n}\right|=1$ and $\left|b_{n}\right|=1$. Our objective here is to show that the rational polarizability expressions in Eqs. 17-20 allow for straightforward and simple analytical predictions of the unitary limit of the electric and magnetic dipoles.

The analysis of the unitary limit is simplified by the use of the so-called $K$-matrix $[29,61,62]$. The reactance $K$-matrix is linked to the $T$-matrix by $K=i T\left(I+T^{-1}\right)$ which allows for casting the $K_{n}^{(e, h)}$ elements with respect to the scattering Mie coefficients:

$$
\begin{aligned}
& \left(a_{n}\right)^{-1}=-i\left(K_{n}^{e}\right)^{-1}+1, \\
& \left(b_{n}\right)^{-1}=-i\left(K_{n}^{h}\right)^{-1}+1 .
\end{aligned}
$$

The unitary limit conditions, $\left|a_{n}\right|=1,\left|b_{n}\right|=1$ lead to $\left(K_{n}^{e}\right)^{-1}=0$ and $\left(K_{n}^{h}\right)^{-1}=0$ respectively. We now have to derive the expressions of the $K$-matrix elements to get the unitary limit. The coefficients can be obtained thanks to the expressions of the Mie coefficients in Eqs. 1a,1b and Eqs. 31a,31b:

$$
\begin{gathered}
K_{n}^{e}=\frac{j_{n}\left(z_{0}\right)}{h_{n}^{+}\left(z_{0}\right)} \frac{\epsilon \varphi_{n}^{1}\left(z_{0}\right)-\varphi_{n}^{1}\left(z_{s}\right)}{\epsilon \varphi_{n}^{2}\left(z_{0}\right)-\varphi_{n}^{1}\left(z_{s}\right)} \\
K_{n}^{h}=\frac{j_{n}\left(z_{0}\right)}{h_{n}^{+}\left(z_{0}\right)} \frac{\varphi_{n}^{1}\left(z_{0}\right)-\varphi_{n}^{1}\left(z_{s}\right)}{\varphi_{n}^{2}\left(z_{0}\right)-\varphi_{n}^{1}\left(z_{s}\right)} .
\end{gathered}
$$

The unitary limit condition corresponds to the cancellation of the denominators in Eqs. 32,33 for the electric and magnetic cases respectively:

$$
\begin{aligned}
\epsilon \varphi_{n}^{2}\left(z_{0}\right)-\varphi_{n}^{1}\left(z_{s}\right) & =0 \\
\varphi_{n}^{2}\left(z_{0}\right)-\varphi_{n}^{1}\left(z_{s}\right) & =0 .
\end{aligned}
$$

We must now find the analytical solutions of these conditions. This objective requires the analytical expressions of functions $\varphi_{n}^{2}\left(z_{0}\right)$ and $\varphi_{n}^{1}\left(z_{s}\right)$. Function $\varphi_{n}^{1}\left(z_{s}\right)$ can be accurately obtained with expressions in Eq. 6 and Eq. 7 for the electric and magnetic case respectively. Regarding function $\varphi_{n}^{2}\left(z_{0}\right)$, for $n=1$, it could be approximated thanks to a Taylor expansion near 0 :

$$
\varphi_{1}^{2}\left(z_{0}\right) \approx-1+z_{0}^{2}-z_{0}^{4}
$$


However, the accuracy of this expansion rapidly decreases as $z_{0}$ increases. This weak convergence is due to the presence of poles in $\varphi_{1}^{2}\left(z_{0}\right)$. This issue can be overcome by expanding the function through a pole form:

$$
\varphi_{n}^{2}(z)=-n+\sum_{m=1}^{\infty} \frac{2 z^{2}}{z^{2}-b_{n, m}^{2}}
$$

where poles $b_{n, m}$ of $\varphi_{n}^{2}(z)$ are complex, one pole is real and the other one is imaginary. In the dipolar order, we can use a pole expansion with a corrective term to improve the accuracy of this pole expansion:

$$
\varphi_{1}^{2}\left(z_{0}\right) \approx-1+\frac{2 z_{0}^{2}}{z_{0}^{2}-b_{1,1}^{2}}+\frac{2 z_{0}^{2}}{z_{0}^{2}-b_{1,2}^{2}}+\frac{2 z_{0}^{2}}{z_{0}^{2}-\Delta_{L}^{2}}
$$

with $b_{1,1}=i 1.199678, b_{1,2}=2.79839$ and $\Delta_{L}=3.85993$.

Expansions of $\varphi_{1}^{1}\left(z_{S}\right)$ provided in Eq. 6 and Eq. 7 for the electric and magnetic cases respectively can be used in Eqs. 34,35 to get the expression of the dielectric permittivities, $\epsilon_{\mathrm{UL}}^{a_{1}}$ and $\epsilon_{\mathrm{UL}}^{b_{1}}$, that maximize the electromagnetic scattering in the electric and magnetic dipolar channels:

$$
\begin{array}{r}
\epsilon_{\mathrm{UL}}^{a_{1}} \approx \frac{-\left(\varphi_{1}^{2}\left(z_{0}\right)+\frac{2 z_{0}^{2}}{r_{0}^{2}}\right) \pm \sqrt{\Delta_{U L}}}{-\frac{2 \varphi_{1}^{2}\left(z_{0}\right) z_{0}^{2}}{r_{1}^{2}}}, \\
\epsilon_{U L}^{b_{1}}=\left(\frac{r_{0}^{2}-\varphi_{1}^{2}\left(z_{0}\right)-1}{r_{0} z_{0}}\right)^{2}
\end{array}
$$

In Eq.39 the expression of $\varphi_{1}^{2}\left(z_{0}\right)$ is taken from Eq. 38 with $\Delta_{U L}=\left(\varphi_{1}^{2}\left(z_{0}\right)+\frac{2 z_{0}^{2}}{r_{0}^{2}}\right)^{2}-8 \frac{\varphi_{1}^{2}\left(z_{0}\right) z_{0}^{2}}{r_{1}^{2}}$. A fundamental difference between these two expressions is that the unitary limit of the electric dipole leads to a second order polynomial equation while the magnetic dipole leads to a $1^{\text {st }}$ order differential equation.

One plots the two solutions of the electric dipole with respect to the parameter size in Fig.5. One observes in Fig. 5 the two dielectric permittivities, solutions of the unitary limit for the electric dipole. The two solutions are purely real, with a positive Fig.5(b) and a negative real part Fig.5(a). Finding two solutions for the electric dipole resonances, one positive and one negative, shows that the sole expression in Eq. 39 catches the LSPR and Mie resonances. This expression is much more accurate than the quasi-static expression of small metallic particles but it can be used in a similar way to predict easily the resonance conditions. For small scatterers with parameter sizes ranging between 0 and 1, Fig. 5 clearly shows that the LSPR can be obtained for metals with dielectric permittivities ranging between $\epsilon=[-4.5 ;-2]$ while Mie resonances span almost all the dielectric permittivities ranging between $\epsilon=[20 ;+\infty]$. For metals, the quasi-static permittivity $\epsilon_{m} \rightarrow-2$ deduced from the Frölich conditions is retrieved for $z_{0} \rightarrow 0$. One observes in Fig.5(b) the unique solution for the magnetic dipole, which confirms that high refractive index materials are the only material that can satisfy this unitary limit condition.

\subsection{Upper bound: Ideal Absorption}

Ideal absorption describes the limit of absorption of the electromagnetic field by a given multipolar order [28]. It is convenient to study the ideal absorption in the framework of the scattering $S$-matrix that links, for each multipolar electric or magnetic component, the outgoing field to the incoming field. Ideal absorption is maximal in this given multipolar channel when the 

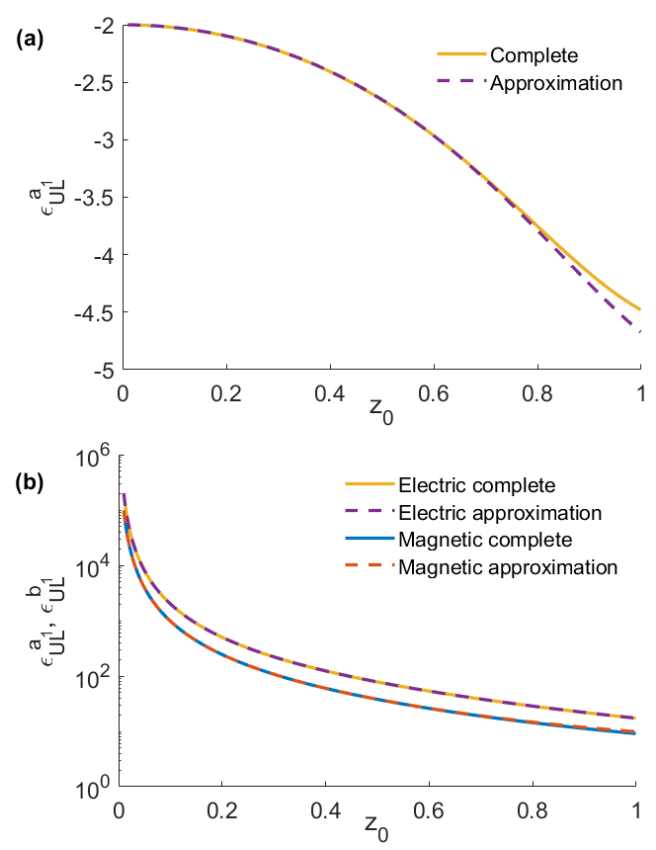

Fig. 5. Dielectric permittivity that satisfies the unitary limit dipolar condition for metals (a) and dielectrics (b). Full lines: complete calculations; electric dipole (yellow) calculated with Eq. 34, magnetic dipole (blue) calculated with Eq. 35. Dotted lines: approximate expressions calculated with Eq. 39 for the electric dipole in (a) and (b) and with Eq. 40 for the magnetic dipole in (b).

electromagnetic field is fully absorbed by the scatterer resulting in the cancellation of the scattered field, requiring $S_{n}^{(e, h)}=0$. The $S$-matrix is linked with the $T$ matrix, $S=I+2 T$, which allows for expanding the $S$-matrix elements with respect to the multipolar Mie coefficients:

$$
\begin{gathered}
S_{n}^{e}=\frac{h_{n}^{-}\left(z_{0}\right)}{h_{n}^{+}\left(z_{0}\right)} \frac{\epsilon \varphi_{n}^{-}\left(z_{0}\right)-\varphi_{n}^{1}\left(z_{s}\right)}{\epsilon \varphi_{n}^{+}\left(z_{0}\right)-\varphi_{n}^{1}\left(z_{s}\right)}, \\
S_{n}^{h}=\frac{h_{n}^{-}\left(z_{0}\right)}{h_{n}^{+}\left(z_{0}\right)} \frac{\varphi_{n}^{-}\left(z_{0}\right)-\varphi_{n}^{1}\left(z_{s}\right)}{\varphi_{n}^{+}\left(z_{0}\right)-\varphi_{n}^{1}\left(z_{s}\right)}
\end{gathered}
$$

The question is now to determine for a given parameter size $z_{0}$ the composition of the scatterer that leads to this ideal absorption condition. For that purpose, we need to solve equations $S_{n}^{(e)}=0=\epsilon \varphi_{n}^{-}\left(z_{0}\right)-\varphi_{n}^{1}\left(z_{s}\right)$ and $S_{n}^{(h)}=0=\varphi_{n}^{-}\left(z_{0}\right)-\varphi_{n}^{1}\left(z_{s}\right)$. Expression of $\varphi_{1}^{-}\left(z_{0}\right)$ is derived in Eq. 10 while expressions of $\varphi_{1}^{1}\left(z_{s}\right)$ are derived in Eq. 6 (electric case) and Eq. 8 (magnetic case). Their use leads to second and first order equations for the electric and magnetic cases respectively whose solutions $\epsilon_{I A}^{(e, h)}$ are:

$$
\begin{aligned}
\epsilon_{I A}^{e} & =\frac{-\left(-i z_{0}+z_{0}^{2}-1+\frac{2 z_{0}^{2}}{r_{0}^{2}}+\frac{2 i z_{0}^{3}}{r_{0}^{2}}\right) \pm \sqrt{\Delta_{I A}}}{2\left(\frac{i z_{0}^{3}}{r_{1}^{2}}-\frac{z_{0}^{4}}{r_{1}^{2}}+\frac{z_{0}^{2}}{r_{1}^{2}}\right)}, \\
\Delta_{I A} & =\left(-i z_{0}+z_{0}^{2}-1+\frac{2 z_{0}^{2}}{r_{0}^{2}}+\frac{2 i z_{0}^{3}}{r_{0}^{2}}\right)^{2}-4\left(-2-2 i z_{0}\right)\left(\frac{i z_{0}^{3}}{r_{1}^{2}}-\frac{z_{0}^{4}}{r_{1}^{2}}+\frac{z_{0}^{2}}{r_{1}^{2}}\right)
\end{aligned}
$$





Fig. 6. Trajectory of the dielectric permittivity, $\epsilon_{I A}^{(e, h)}$, that satisfies the ideal absorption condition (full solid lines) and the pole (dashed lines) for the electric (e) and magnetic (h) dipolar cases in the $\mathbb{R} e(\epsilon)$ and $\mathbb{I} m(\epsilon)$ plane for $\left.\left.z_{0}=\right] 0 ; 1\right]$. (a) Dielectric scatterers, (b) metallic scatterers.

$$
\epsilon_{I A}^{h}=\frac{\left(-r_{0}^{2}+z_{0}^{2}-i r_{0}^{2} z_{0}\right)^{2}}{\left(r_{0} z_{0}+i r_{0} z_{0}^{2}\right)^{2}} .
$$

We plot in Fig. 6 the trajectory of the solutions, $\epsilon_{I A}^{(e, h)}$, in the $\mathbb{R} e(\epsilon)$ and $\mathbb{I} m(\epsilon)$ plane while the parameter size spans the range ]0; 1]. For the sake of comparison, the poles obtained in Eq. 26 and 27 are superimposed on this figure. We first remark that the trajectories of the solutions of ideal absorption and poles are perfectly symmetric with respect to the real axis $\mathbb{R} e(\epsilon)$ : dielectric permittivities solutions of poles and ideal absorption are complex conjugates. This result can be simply predicted by comparing equations $\epsilon \varphi_{1}^{+}\left(z_{0}\right)-\varphi_{1}^{1}\left(z_{s}\right)=0$ and $\epsilon \varphi_{1}^{-}\left(z_{0}\right)-\varphi_{1}^{1}\left(z_{s}\right)=0$ that need to be solved for the ideal absorption and poles respectively: they differ only from the sign of the imaginary terms in $\varphi_{1}^{ \pm}\left(z_{0}\right)= \pm i z_{0}-\frac{1}{1 \mp i z_{0}}$. This result highlights the link between coherent perfect absorption (CPA) and time-reversed lasers [63] since taking $\operatorname{Im}(\epsilon) \rightarrow-\operatorname{Im}(\epsilon)$ corresponds to $t \rightarrow-t$. Ideal absorption, or coherent perfect absorption, can be retrieved by the poles of the scatterers and taking the complex conjugate.

A second remark when observing Fig. 6 is that the imaginary parts for morphological and plasmonics cases are very similar in the case of the electric dipole. A third remark is that losses required to achieve ideal absorption are significantly smaller for electric dipoles than for magnetic dipoles for dielectric permittivities higher than 40. The asymptotic limits of functions $\operatorname{Im}\left(\epsilon_{I A}^{(e)}\right)$ and $\operatorname{Im}\left(\epsilon_{I A}^{(h)}\right)$ (Eqs. 41a,41b) when $z_{0} \rightarrow 0$ are very different. It turns out that electric dipoles can achieve ideal absorption in subwavelength sized scatterers composed of high dielectric permittivity $(\epsilon>40)$ with extremely weak losses, while magnetic dipoles require lower losses for achieving ideal absorption for smaller dielectric permittivities.

\subsection{Summary of the approximate expressions}

We summarize the different expressions obtained in Tables 1 and 2. In these expressions, $r_{0}=3.14$ and $r_{1}=4.49, q_{1}=3.14$ for dielectrics and $q_{1}=2.55$ for metals. For the expression 
of the Unitary Limit, we remind that $\Delta_{U L}=\left(\varphi_{1}^{2}\left(z_{0}\right)+\frac{2 z_{0}^{2}}{r_{0}^{2}}\right)^{2}-8 \frac{\varphi_{1}^{2}\left(z_{0}\right) z_{0}^{2}}{r_{1}^{2}}$ and that function $\varphi_{1}^{2}\left(z_{0}\right)$ can be approximated with:

$$
\varphi_{1}^{2}\left(z_{0}\right) \approx-1+\frac{2 z_{0}^{2}}{z_{0}^{2}-b_{1,1}^{2}}+\frac{2 z_{0}^{2}}{z_{0}^{2}-b_{1,2}^{2}}+\frac{2 z_{0}^{2}}{z_{0}^{2}-\Delta_{L}^{2}},
$$

with $b_{1,1}=i 1.199678, b_{1,2}=2.79839$ and $\Delta_{L}=3.85993$. For the ideal absorption, $\Delta_{I A}=$ $\left(-i z_{0}+z_{0}^{2}-1+\frac{2 z_{0}^{2}}{r_{0}^{2}}+\frac{2 i z_{0}^{3}}{r_{0}^{2}}\right)^{2}-4\left(-2-2 i z_{0}\right)\left(\frac{i z_{0}^{3}}{r_{1}^{2}}-\frac{z_{0}^{4}}{r_{1}^{2}}+\frac{z_{0}^{2}}{r_{1}^{2}}\right)$ and in the case of the anapole, $\Delta_{z}=\left(\frac{-\epsilon}{5}-\frac{2 \epsilon^{2}}{r_{1}^{2}}+\frac{2 \epsilon}{r_{0}^{2}}\right)^{2}-4(2 \epsilon-2)\left(\frac{\epsilon^{2}}{5 r_{1}^{2}}\right)$.

Table 1. Electric dipolar mode

\begin{tabular}{|c|c|}
\hline \multirow{2}{*}{ Dipolar Mie coefficient } & $\epsilon\left(2-\frac{z_{0}^{2}}{5}\right)-2 \frac{1-\left(\frac{z s}{q 1}\right)^{2}}{1-\left(\frac{z s}{r_{1}}\right)^{2}}$ \\
\hline & $a_{1}\left(z_{0}\right)=\overline{3\left(z_{0}+i\right) e^{i z_{0}\left(1+\frac{z_{0}^{2}}{10}\right)}} \overline{\epsilon\left(i z_{0}-\frac{1}{1-i z_{0}}\right)-2 \frac{1-\left(\frac{z_{s}}{q_{1}}\right)^{2}}{1-\left(\frac{z_{s}}{r_{1}}\right)^{2}}}$ \\
\hline Poles & $\begin{array}{c}\epsilon_{p, e}^{2}\left(-i \frac{z_{0}^{3}}{r_{1}^{2}}-\frac{z_{0}^{4}}{r_{1}^{2}}+\frac{z_{0}^{2}}{r_{1}^{2}}\right)+\epsilon_{p, e}\left(i z_{0}+z_{0}^{2}-1\right. \\
\left.+\frac{2 z_{0}^{2}}{r_{0}^{2}}-2 i \frac{z_{0}^{3}}{r_{0}^{2}}\right)+\left(2 i z_{0}-2\right)=0\end{array}$ \\
\hline Zero (anapole) & $\epsilon_{z_{0}}=\frac{-\left(2-\frac{z_{0}^{2}}{5}+\frac{z_{0}^{2}}{r_{0}^{2}}\right)+\sqrt{\Delta_{z}}}{2\left(\frac{-2 z_{0}^{2}}{r_{1}^{2}}+\frac{z_{0}^{4}}{r_{1}^{2}}\right)}$ \\
\hline Unitary limit & $\epsilon_{\mathrm{UL}}^{a_{1}} \approx \frac{-\left(\varphi_{1}^{2}\left(z_{0}\right)+\frac{2 z_{0}^{2}}{r_{0}^{2}}\right) \pm \sqrt{\Delta_{U L}}}{-\frac{2 \varphi_{1}^{2}\left(z_{0}\right) z_{0}^{2}}{r_{1}^{2}}}$ \\
\hline Ideal absorption & $\epsilon_{I A}^{e}=\frac{-\left(-i z_{0}+z_{0}^{2}-1+\frac{2 z_{0}^{2}}{r_{0}^{2}}+\frac{2 i z_{0}^{3}}{r_{0}^{2}}\right) \pm \sqrt{\Delta_{I A}}}{2\left(\frac{i z_{0}^{3}}{r_{1}^{2}}-\frac{z_{0}^{4}}{r_{1}^{2}}+\frac{z_{0}^{2}}{r_{1}^{2}}\right)}$ \\
\hline
\end{tabular}

Table 2. Magnetic dipolar mode

\begin{tabular}{cc}
\hline Mie coefficients & $b_{1}\left(z_{0}\right)=\frac{-z_{0}^{3}}{3\left(z_{0}+i\right) e^{i z_{0}}\left(1+\frac{z_{0}^{2}}{10}\right)} \frac{3-\frac{z_{0}^{2}}{5}+r_{0}\left(z_{s}-r_{0}\right)}{\left(i z_{0}-\frac{1}{1-i z_{0}}\right)+1+r_{0}\left(z_{s}-r_{0}\right)}$ \\
\hline Poles & $\epsilon_{p, h}=\left(\frac{-z_{0}^{2}+r_{0}^{2}-i r_{0}^{2} z_{0}}{r_{0} z_{0}-i r_{0} z_{0}^{2}}\right)^{2}$ \\
\hline Unitary limit & $\epsilon_{U L}^{b_{1}}=\left(\frac{r_{0}^{2}-\varphi_{1}^{2}\left(z_{0}\right)-1}{r_{0} z_{0}}\right)^{2}$ \\
\hline Ideal absorption & $\epsilon_{I A}^{h}=\frac{\left(-r_{0}^{2}+z_{0}^{2}-i r_{0}^{2} z_{0}\right)^{2}}{\left(r_{0} z_{0}+i r_{0} z_{0}^{2}\right)^{2}}$
\end{tabular}

\section{Conclusion}

To conclude, we used approximate expressions of Mie coefficients to get the analytical expressions of eigen-values and physical bounds of dipolar LSPR and Mie resonances of subwavelength sized scatterers. The same expressions of Mie coefficients are used successively to retrieve the dipolar and quadrupolar resonances of scatterers and to find the analytical expressions of the poles, anapoles, unitary limit and ideal absorption. The results obtained with approximate expressions are always compared with full numerical results provided by the complete Mie theory. 
Approximate expressions allow the finding of the first two electric and magnetic eigen-frequencies, a fundamental mode characterized by a low quality factor associated with a weak dependence on the dielectric permittivity and a first mode characterized by a much better quality factor and a much stronger dependence on the dielectric permittivity. The eigen-modes of the scatterer can also be expanded in term of the complex dielectric permittivity. The analytical expressions provide one solution for the magnetic dipolar case, with a positive real part of the dielectric permittivity, and two solutions for the electric dipolar case, with a positive and a negative real part of the dielectric permittivity. Finding two solutions with the same approximate expression of the electric Mie coefficient extends the so-called Frölich conditions to both metallic and dielectric scatterers. Solutions of the ideal absorption condition are complex conjugates of dielectric permittivities found for the pole solutions. This result nicely illustrates the fact that the ideal absorption condition, also called coherent perfect absorption, corresponds to a time-reversed laser. We also showed that for small parameter sizes (typically smaller than 0.5 ), ideal absorption can be achieved through electric dipoles with real dielectric permittivities typically higher than 40 with extremely small losses.

These approximate expressions also provide real solutions for achieving the physical bounds, minimum and maximum. The minimum of light scattering is studied in the electric case (electric anapole) and we find a real solution for dielectric permittivities higher typically than 6.96, meaning that a complete cancellation of the electric dipolar coefficient can be observed in the spectrum for any scatterer with a dielectric permittivity higher than this value (in air or vacuum). On the other side, the maximum of light scattering is obtained for both positive and negative real dielectric permittivities in the electric case and for positive dielectric permittivities for the magnetic case. Finally, this work shows how simple and accurate expressions of the Mie coefficients can be used to accurately and simply predict some of the most interesting features of electromagnetic resonators, including the calculation of complex eigen-frequencies, coherent perfect absorption, minimum and maximum scattering bounds.

\section{Acknowledgments}

The authors acknowledge the support of Aix-Marseille Université and Direction Générale des Armées. The authors thank Philippe Pouliguen and Rémi Colom for fruitful discussions.

\section{Disclosures}

The authors declare no conflicts of interest.

\section{References}

1. S. Maier, Plasmonics: Fundamentals and Applications (Springer, 2007).

2. W. A. Murray and W. L. Barnes, "Plasmonic materials," Adv. materials 19, 3771-3782 (2007).

3. S. Enoch and N. Bonod, Plasmonics: From Basics to Advanced Topics (Springer, Berlin, 2012).

4. M. I. Stockman, K. Kneipp, S. I. Bozhevolnyi, S. Saha, A. Dutta, J. Ndukaife, N. Kinsey, H. Reddy, U. Guler, V. M. Shalaev et al., "Roadmap on plasmonics," J. Opt. 20, 043001 (2018).

5. A. Moroz, "Depolarization field of spheroidal particles," JOSA B 26, 517-527 (2009).

6. A. Wokaun, J. Gordon, and P. Liao, "Radiation damping in surface-enhanced raman scattering," Phys. Rev. Lett. 48, 957 (1982).

7. D. Schebarchov, B. Auguié, and E. C. Le Ru, "Simple accurate approximations for the optical properties of metallic nanospheres and nanoshells," Phys. Chem. Chem. Phys. 15, 4233-4242 (2013).

8. A. I. Kuznetsov, A. E. Miroshnichenko, M. L. Brongersma, Y. S. Kivshar, and B. Luk'yanchuk, "Optically resonant dielectric nanostructures," Science. 354, aag2472 (2016).

9. M. Decker and I. Staude, "Resonant dielectric nanostructures: a low-loss platform for functional nanophotonics," J. Opt. 18, 103001 (2016).

10. A. Krasnok, M. Caldarola, N. Bonod, and A. Alú, "Spectroscopy and biosensing with optically resonant dielectric nanostructures," Adv. Opt. Mater. 6, 1701094 (2018).

11. Q. Zhao, J. Zhou, F. Zhang, and D. Lippens, "Mie resonance-based dielectric metamaterials," Mater. Today 12, 60-69 (2009). 
12. G. Boudarham, R. Abdeddaim, and N. Bonod, "Enhancing the magnetic field intensity with a dielectric gap antenna," Appl. Phys. Lett. 104, 021117 (2014).

13. M. A. Moussu, L. Ciobanu, S. Kurdjumov, E. Nenasheva, B. Djemai, M. Dubois, A. G. Webb, S. Enoch, P. Belov, R. Abdeddaim et al., "Systematic analysis of the improvements in magnetic resonance microscopy with ferroelectric composite ceramics," Adv. materials 31, 1900912 (2019).

14. R. E. Jacobsen, A. V. Lavrinenko, and S. Arslanagić, "Water-based metasurfaces for effective switching of microwaves," IEEE Antennas Wirel. Propag. Lett. 17, 571-574 (2018).

15. R. Gomez-Medina, B. Garcia-Camara, I. Suarez-Lacalle, F. Gonzalez, F. Moreno, M. Nieto-Vesperinas, and J. J. Saenz, "Electric and magnetic dipolar response of germanium nanospheres: interference effects, scattering anisotropy, and optical forces,' J. Nanophotonics 5, 053512 (2011).

16. A. B. Evlyukhin, C. Reinhardt, and B. N. Chichkov, "Multipole light scattering by nonspherical nanoparticles in the discrete dipole approximation,” Phys. Rev. B: Condens. Matter Mater. Phys. 84, 235429 (2011).

17. A. García-Etxarri, R. Gómez-Medina, L. S. Froufe-Pérez, C. López, L. Chantada, F. Scheffold, J. Aizpurua, M. Nieto-Vesperinas, and J. J. Sáenz, "Strong magnetic response of submicron silicon particles in the infrared," Opt. express 19, 4815-4826 (2011).

18. D. Tzarouchis and A. Sihvola, "Light scattering by a dielectric sphere: perspectives on the mie resonances," Appl. Sci. 8, 184 (2018).

19. V. E. Babicheva and A. B. Evlyukhin, "Analytical model of resonant electromagnetic dipole-quadrupole coupling in nanoparticle arrays,” Phys. Rev. B 99, 195444 (2019).

20. H. Hulst, Light scattering by small particles, Structure of matter series (Wiley, 1957).

21. J. Geffrin, B. García-Cámara, R. Gómez-Medina, P. Albella, L. Froufe-Pérez, C. Eyraud, A. Litman, R. Vaillon, F. González, M. Nieto-Vesperinas et al., "Magnetic and electric coherence in forward-and back-scattered electromagnetic waves by a single dielectric subwavelength sphere," Nat. communications 3, 1171 (2012).

22. B. Rolly, J.-M. Geffrin, R. Abdeddaim, B. Stout, and N. Bonod, "Controllable emission of a dipolar source coupled with a magneto-dielectric resonant subwavelength scatterer,' Sci. reports 3 (2013).

23. A. E. Krasnok, D. S. Filonov, C. R. Simovski, Y. S. Kivshar, and P. A. Belov, "Experimental demonstration of superdirective dielectric antenna," Appl. Phys. Lett. 104 (2014).

24. A. Devilez, X. Zambrana-Puyalto, B. Stout, and N. Bonod, "Mimicking localized surface plasmons with dielectric particles,” Phys. Rev. B 92, 241412 (2015).

25. D. Bedeaux and P. Mazur, "On the critical behaviour of the dielectric constant for a nonpolar fluid," Physica 67, 23-54 (1973).

26. L. Novotny and B. Hecht, Principles of Nano-Optics (Cambridge University Press, 2006).

27. S. Albaladejo, R. Gómez-Medina, L. Froufe-Pérez, H. Marinchio, R. Carminati, J. Torrado, G. Armelles, A. GarcíaMartín, and J. J. Saénz, "Radiative corrections to the polarizability tensor of an electrically small anisotropic dielectric particle,” Opt. Express 18, 3556-3567 (2010).

28. V. Grigoriev, N. Bonod, J. Wenger, and B. Stout, "Optimizing nanoparticle designs for ideal absorption of light," ACS photonics 2, 263-270 (2015).

29. R. Colom, A. Devilez, N. Bonod, and B. Stout, "Optimal interactions of light with magnetic and electric resonant particles," Phys. Rev. B 93, 045427 (2016).

30. L. Tsang and J. A. Kong, "Multiple scattering of electromagnetic waves by random distributions of discrete scatterers with coherent potential and quantum mechanical formalism," J. Appl. Phys. 51, 3465-3485 (1980).

31. R. Colom, A. Devilez, S. Enoch, B. Stout, and N. Bonod, "Polarizability expressions for predicting resonances in plasmonic and mie scatterers," Phys. Rev. A 95, 063833 (2017).

32. M. Abramowitz and I. A. Stegun, Handbook of mathematical functions: with formulas, graphs, and mathematical tables, vol. 55 (Courier Corporation, 1965).

33. G. Watson, A Treatise on the Theory of Bessel Functions, Cambridge mathematical library (Cambridge University Press, 1944).

34. G. A. Baker, G. A. Baker Jr, G. Baker, P. Graves-Morris, and S. S. Baker, Pade Approximants: Encyclopedia of Mathematics and It's Applications, Vol. 59 George A. Baker, Jr., Peter Graves-Morris, vol. 59 (Cambridge University Press, 1996).

35. C. M. Bender and S. A. Orszag, Advanced mathematical methods for scientists and engineers I: Asymptotic methods and perturbation theory (Springer Science \& Business Media, 2013).

36. P. Johnson and R.-W. Christy, "Silver (ag)," Opt. Constants Noble Met. Phys. Rev. B 6, 4370-4379 (1972).

37. I. B. ZelD́ovich, "Electromagnetic interaction with parity violation,” Sov. Phys. JETP 6, 1184-1186 (1958).

38. G. Afanasiev and V. Dubovik, "Some remarkable charge-current configurations,” Phys. Part. Nucl. 29, 891-945 (1998)

39. B. Luk'yanchuk, R. Paniagua-Domínguez, A. I. Kuznetsov, A. E. Miroshnichenko, and Y. S. Kivshar, "Suppression of scattering for small dielectric particles: anapole mode and invisibility," Philos. Transactions Royal Soc. A: Math. Phys. Eng. Sci. 375, 20160069 (2017).

40. V. Valuckas, R. Paniagua-Domínguez, Y. H. Fu, B. Luk’yanchuk, and A. I. Kuznetsov, "Direct observation of resonance scattering patterns in single silicon nanoparticles," Appl. Phys. Lett. 110, 091108 (2017).

41. S. E. Svyakhovskiy, V. V. Ternovski, and M. I. Tribelsky, "Anapole: Its birth, life, and death,” Opt. express 27, 23894-23904 (2019). 
42. R. Colom, R. McPhedran, B. Stout, and N. Bonod, "Modal analysis of anapoles, internal fields, and fano resonances in dielectric particles,” J. Opt. Soc. Am. B 36, 2052-2061 (2019).

43. H. Lamb, "On a peculiarity of the wave-system due to the free vibrations of a nucleus in an extended medium," Proc. Lond. Math. Soc. 1, 208-213 (1900).

44. V. Grigoriev, A. Tahri, S. Varault, B. Rolly, B. Stout, J. Wenger, and N. Bonod, "Optimization of resonant effects in nanostructures via weierstrass factorization," Phys. Rev. A 88, 011803 (2013).

45. C. Sauvan, J.-P. Hugonin, I. Maksymov, and P. Lalanne, "Theory of the spontaneous optical emission of nanosize photonic and plasmon resonators," Phys. Rev. Lett. 110, 237401 (2013).

46. R. Colom, R. C. McPhedran, B. Stout, and N. Bonod, "Modal expansion of the scattered field: Causality, nondivergence, and nonresonant contribution,” Phys. Rev. B 98, 005400 (2018).

47. P. Lalanne, W. Yan, K. Vynck, C. Sauvan, and J.-P. Hugonin, "Light interaction with photonic and plasmonic resonances," Laser \& Photonics Rev. 12, 1700113 (2018).

48. H. Ammari, A. Dabrowski, B. Fitzpatrick, P. Millien, and M. Sini, "Subwavelength resonant dielectric nanoparticles with high refractive indices," Math. Methods Appl. Sci. 42, 6567-6579 (2019).

49. H. Ammari, P. Millien, and A. L. Vanel, "Modal expansion for plasmonic resonators in the time domain," arXiv preprint arXiv:2003.09200 (2020).

50. P. Y. Chen, D. J. Bergman, and Y. Sivan, “Generalizing normal mode expansion of electromagnetic green's tensor to open systems,” Phys. Rev. Appl. 11, 044018 (2019).

51. Y. Ivanenko, M. Gustafsson, and S. Nordebo, "Optical theorems and physical bounds on absorption in lossy media," Opt. Express 27, 34323-34342 (2019).

52. H. Zhang, C. W. Hsu, and O. D. Miller, "Scattering concentration bounds: brightness theorems for waves," Optica 6, 1321-1327 (2019).

53. M. Gustafsson, K. Schab, L. Jelinek, and M. Capek, "Upper bounds on absorption and scattering," New J. Phys. (2020).

54. H. Noh, Y. Chong, A. D. Stone, and H. Cao, "Perfect coupling of light to surface plasmons by coherent absorption," Phys. review letters 108, 186805 (2012).

55. É. Castanié, R. Vincent, R. Pierrat, and R. Carminati, "Absorption by an optical dipole antenna in a structured environment," Int. J. Opt. 2012 (2012).

56. A. Sentenac, P. Chaumet, and G. Leuchs, "Total absorption of light by a nanoparticle: an electromagnetic sink in the optical regime," Opt. letters 38, 818-820 (2013).

57. S. Tretyakov, "Maximizing absorption and scattering by dipole particles," Plasmonics 9, 935-944 (2014).

58. K. Pichler, M. Kühmayer, J. Böhm, A. Brandstötter, P. Ambichl, U. Kuhl, and S. Rotter, "Random anti-lasing through coherent perfect absorption in a disordered medium," Nature 567, 351-355 (2019).

59. E. Sakat, L. Wojszvzyk, J.-J. Greffet, J.-P. Hugonin, and C. Sauvan, "Enhancing light absorption in a nanovolume with a nanoantenna: Theory and figure of merit," ACS Photonics 7, 1523-1528 (2020).

60. H. Shim, Z. Kuang, and O. D. Miller, “Optical materials for maximal nanophotonic response,” Opt. Mater. Express 10, 1561-1585 (2020).

61. E. C. Le Ru, W. R. Somerville, and B. Auguié, "Radiative correction in approximate treatments of electromagnetic scattering by point and body scatterers," Phys. Rev. A 87, 012504 (2013).

62. T. Raziman, W. Somerville, O. J. Martin, and E. Le Ru, "Accuracy of surface integral equation matrix elements in plasmonic calculations," JOSA B 32, 485-492 (2015).

63. Y. Chong, L. Ge, H. Cao, and A. D. Stone, "Coherent perfect absorbers: time-reversed lasers," Phys. review letters 105, 053901 (2010). 